\title{
Effects of Fructus Ligustri Lucidi Extract on Bone Turnover and Calcium Balance in Ovariectomized Rats
}

\author{
Yan Zhang, ${ }^{a, b}$ Wan-Ping LaI,${ }^{b, c}$ Ping-Chung Leung, ${ }^{d}$ Chun-Fu Wu, ${ }^{a}$ Xin-Sheng YAO, ${ }^{e}$ and \\ Man-Sau WonG $*, b, c$ \\ ${ }^{a}$ Department of Pharmacology, Shenyang Pharmaceutical University; Shenyang, 110016, China: ${ }^{b}$ State Key Laboratory of \\ Chinese Medicine and Molecular Pharmacology; Shenzhen, China: ${ }^{c}$ Open Laboratory of Chirotechnology of the Institute \\ of Molecular Technology for Drug Discovery and Synthesis, Department of Applied Biology and Chemical Technology, the \\ Hong Kong Polytechnic University; Hung Hom, Kowloon, Hong Kong SAR, China: ${ }^{d}$ Department of Orthopaedics and \\ Traumatology, the Chinese University of Hong Kong; Hong Kong SAR, China: and ${ }^{e}$ Joint Laboratory of Biotechnology \\ and Drug Discovery, Graduate School at Shenzhen, Tsinghua University; Shenzhen, 518055, China.
}

Received September 30, 2005; accepted November 23, 2005

The aim of this study was to evaluate the effect of Fructus Ligustri Lucidi (FLL), a kidney-tonifying Chinese herbal medicine, on the biochemical markers of bone turnover, calcium metabolism and balance in osteoporotic rat model developed by ovariectomy. Four weeks after surgical operation, animals were randomly assigned to one of the four treatments for 14 weeks: sham-operated control treated with vehicle (sham, $n=8)$, ovariectomized group treated with vehicle $(\mathrm{OVX}, n=8)$, OVX group treated with $17 \beta$-estradiol $\left(\mathrm{E}_{2}, n=10,2 \mu \mathrm{g} / \mathrm{kg} / \mathrm{d}\right)$ and $\mathrm{OVX}$ group treated with FLL extracts (FLL, $n=10,550 \mathrm{mg} / \mathrm{kg} / \mathrm{d})$. Serum osteocalcin and urinary deoxypyridinoline levels were upregulated in rats in response to $\mathrm{OVX}$, suggesting that the bone turnover rate was accelerated in these animals. Treatment of OVX rats with FLL extract could prevent OVX-induced increase in bone turnover by suppression of both serum osteocalcin $(p<0.05$, vs. OVX) and urinary deoxypyridinoline $(p<0.05, v s$. OVX) levels. In addition, FLL extract could prevent $O V X$-induced loss of calcium in rats by increasing the intestinal calcium absorption rate $(p<0.01, v s$. OVX), suppressing urinary Ca excretion $(p<0.05$, vs. OVX) as well as increasing bone calcium content $(p<0.05$, vs. OVX). Our study is the first to report that FLL can modulate bone turnover and calcium balance in OVX rats and it might be a potential candidate for prevention and treatment of postmenopausal osteoporosis.

Key words Fructus Ligustri Lucidi; ovariectomy; bone turnover; calcium balance

Postmenopausal osteoporosis is a major age-related health problem for women who often have negative calcium balance due to decrease in intestinal calcium absorption, insufficient dietary calcium intake, as well as increase in urinary $\mathrm{Ca}$ loss associated with estrogen deficiency during menopause. ${ }^{1)}$ Hormone replacement therapy (HRT) has been an established regime for prevention of postmenopausal bone loss, ${ }^{2,3)}$ but recent evidence indicates that its long-term use is accompanied by side effects, such as the increased risk of breast, ovarian and endometrial cancer. ${ }^{4,5)}$ Thus, alternative means of proven efficacy and safety should be developed for prevention and treatment of postmenopausal osteoporosis.

Traditional Chinese Medicine (TCM) has been widely used for thousands of years to treat fractures and joint diseases. Many herbs shown to have kidney-tonifying activities are used in TCM formulas for prevention and treatment of osteoporosis. ${ }^{6)}$ Although these herbal medicines are seen as cost-effective alternatives by their traditional users, their international acceptance as a major regimen for prevention and treatment of osteoporosis would require extensive research using modern science.

The source of the crude drug, Fructus Ligustri Lucidi (FLL, Chinese name, Nvzhenzi), is the fruit of Ligustrum lucidum Ait. ${ }^{7)}$ It has been used in traditional Chinese medicine for over 1000 years, mainly to treat such ailments as menopausal problems, blurred vision, tinnitus, rheumatic pains, palpitations, backache, insomnia as well as to alleviate age-related symptoms. $^{8-10)}$ It was first described in the Chinese Materia Medica, Shen Nong Ben Cao Jing (Anonymous, $c a$. 200 B.C). In the theory of traditional Chinese med- icine, it has the effects of maintaining healthy energy, and nourishing the liver and kidneys, is therefore a commonly prescribed herbal material in a number of formulae used to tonify the kidneys and strengthen bone. ${ }^{11,12)}$ Modern research has shown that FLL is useful for prevention of bone marrow loss in cancer patients receiving chemotherapy. ${ }^{13)}$ Hao et al. observed that FLL induced ultrastructural changes on the corticotrophs of rat pituitary gland and provided morphological evidence for the action of FLL in modulating endocrine function. ${ }^{14)}$ However, the beneficial effect on bone and mineral metabolism has not been scientifically evaluated.

In this study, we investigated the effects of the crude FLL extract on bone and mineral metabolism, bone turnover marker and calcium balance in osteoporotic rats. The results provide evidence of the osteoprotective effects of this crude extract in ovariectomized rats.

\section{MATERIALS AND METHODS}

Measurement of Oleanolic Acid in Fructus Ligustri Lucidi (FLL) Extract Dried extract powder of Fructus Ligustri Lucidi was purchased from 999 Company (Shenzhen, China), and the yield of the extract from original plant was $42 \%$. A voucher specimen was deposited in the Shenzhen Research Center of Traditional Chinese Medicine and Natural Products (Shenzhen, P.R.C.), where oleanolic acid was supplied. The content of oleanolic acid in FLL is often used as one of criteria to evaluate the quality of this crude drug.

Contrast solution was prepared by dissolving $10 \mathrm{mg}$ 
oleanolic acid in $10 \mathrm{ml}$ of methanol in volumetric flask. Onegram samples of FLL extract were accurately weighed and treated with chloroform for $2 \mathrm{~h}$. The distilled solution was dried and the residue was dissolved in methanol, then the sample solution was prepared as with the contrast solution. A $10 \mu \mathrm{l}$ solution was subjected to HPLC (LC-10ATVP, SHIMADZU) and analyzed with an UV detector (SPD-M10AVP, SHIMADZU) at $220 \mathrm{~nm}$. The separating conditions were as follows: column, Shim-pack VP-ODS $(4.6 \times 250 \mathrm{~mm}$, ID, $5 \mu \mathrm{m})$; column temperature, $30^{\circ} \mathrm{C}$; mobile phase, methanol : water:acetic acid $(87: 13: 0.1, \mathrm{~V} / \mathrm{V} / \mathrm{V})$; and flow rate, $1.0 \mathrm{ml} / \mathrm{min}$. The content of oleanolic acid in the FLL extracts was calculated from the relevant peak areas with external standard method.

Animals and Treatments Thirty-six 3-month-old virgin female Sprague-Dawley rats weighing about $225 \mathrm{~g}$ were purchased from Guangzhou University of Traditional Chinese Medicine (Guangzhou, China). Rats were housed in a room with alternating $12 \mathrm{~h}$ periods of light and dark, ambient temperature of $23 \pm 1^{\circ} \mathrm{C}$ and humidity of $55 \pm 5 \%$. All animals were allowed free access to distilled water and fed on a commercial diet. All animals were weighed weekly during the experimental period.

The acclimatized rats underwent either bilateral laparotomy (sham, $n=8$ ) or bilateral ovariectomy (OVX, $n=28$ ). Four weeks after recovering from surgery, the OVX rats were randomly divided into three groups: vehicle-treated (OVX, $n=8)$; estrogen-treated $\left(\mathrm{E}_{2}, n=10,2 \mu \mathrm{g} / \mathrm{kg} / \mathrm{d}\right)$; FLL extracttreated (FLL, $n=10,550 \mathrm{mg} / \mathrm{kg} / \mathrm{d}$ ). Powdered extract of FLL was dissolved in $\mathrm{ddH}_{2} \mathrm{O}$ and was orally administered to rats at the dosage based on the adult dose from clinical trials and calculated using the dose converting table between man and rat. Rats in the sham and vehicle-treated OVX groups were given distilled water based on the body weight $(1 \mathrm{ml}$ distilled water $/ 100 \mathrm{~g}$ body weight). The amount of diet administered to each group was based on the group with minimum average intake as calculated based on ad libitum feeding during the acclimation period.

Before sacrifice, each rat was individually housed in a metabolic cage and $24 \mathrm{~h}$ urine and feces samples were collected using separators. The residual food and the feces were weighed. The urine samples were acidified with $2 \mathrm{ml} 1 \mathrm{~mol} / \mathrm{l}$ $\mathrm{HCl}$ and stored at $-20^{\circ} \mathrm{C}$ until they were assayed. At sacrifice, blood was taken from abdominal aorta under light ether anesthesia; serum was then prepared by centrifugation of the collected blood (2000 rpm for $20 \mathrm{~min}$ ) and stored at $-80^{\circ} \mathrm{C}$ for biochemical analyses. The uterus was separated from the surrounding adipose and connective tissues and its wet weight was determined. The tibias were dissected out and cleaned of all soft tissue, then wrapped in saline-soaked towels, sealed in plastic bags and stored $-20^{\circ} \mathrm{C}$ for further analysis. All studies were conducted according to the principles and procedures contained in the most recent publication of the NIH Guide for the Care and Use of Laboratory Animals National Research Council. ${ }^{15}$

Serum Chemistry Serum calcium and phosphorus concentrations were measured by standard colorimetric methods using an automatic analyzer, ALCYON 300i (Abbott Laboratories Ltd., U.S.A.). The kits were purchased from Zhongsheng Beikong Bio-technology and Science Inc. (Beijing, China). Serum osteocalcin level was determined using sand- wich ELISA kit (Biomedical Technologies Inc., Stoughton, MA, U.S.A.) for rat osteocalcin only.

Urine Chemistry The concentration of calcium, phosphorus and creatinine in urine was measured by the same method used for the serum samples. Urine deoxypyridinoline was assayed by competitive enzyme immunoassay using METRA $^{\text {TM }}$ DPD EIA kit (Quidel Corporation, San Diego, CA, U.S.A.). Measurements for calcium, phosphorus and DPD were corrected for urinary concentration by creatinine.

Bone Calcium Content The left tibias were dried at $120^{\circ} \mathrm{C}$ for $6 \mathrm{~h}$ in a muffle furnace and the dry weights were recorded. The bones were then incinerated at $800^{\circ} \mathrm{C}$ for $16 \mathrm{~h}$ and the ash weighed. One hundred milligrams of bone ash was then dissolved in $2 \mathrm{ml}$ of $37 \% \mathrm{HCl}$ and diluted with Millin-Q water. The calcium content was determined by atomic absorption spectrophotometry (PerkinElmer, AAnalyst 100 Spectrometer).

Calcium Balance Study Feces were dried at $120^{\circ} \mathrm{C}$ and milled; the powdered feces was then dissolved in $6 \mathrm{M} \mathrm{HCl}$, warmed to $\mathrm{ca} .80^{\circ} \mathrm{C}$ for $10 \mathrm{~min}$. Fecal calcium content was measured by a colorimetric method using a kit similar to that for analyzing serum samples. Calcium intake was estimated from daily food intake. The absorption and retention rates of calcium were calculated by the following equations ${ }^{16)}$ : Ca absorption rate $(\%)=(\mathrm{Ca}$ intake - fecal $\mathrm{Ca}) / \mathrm{Ca}$ intake $\times 100 ; \mathrm{Ca}$ balance $=\mathrm{Ca}$ intake $-($ urinary $\mathrm{Ca}+$ fecal $\mathrm{Ca})$; $\mathrm{Ca}$ retention rate $(\%)=\mathrm{Ca}$ balance $/ \mathrm{Ca}$ intake $\times 100$.

Statistical Analysis Data are expressed as mean values S.E.M. One-way ANOVA was used to compare data from all groups and Turkey's test used as post test after ANOVA was performed to compare pairs of groups by the statistical software of GraphPad PRISM (Version 4.0). A $p$ value of less than 0.05 was considered statistically significant.

\section{RESULTS}

Quality Assessment of FLL Extract Oleanolic acid is a commonly used marker for authentication of FLL according to the Chinese Pharmacopoeia. ${ }^{7)}$ Figure 1A is a typical chromatography profile in which oleanolic acid standard showed a high peak at retention time of $22.5 \mathrm{~min}$. A typical HPLC chromatogram profile of the FLL extract is shown in Fig. 1B. Oleanolic acid was adequately resolved from other unknown compounds and could be clearly identified, confirming the identity of the FLL extract. The amount of oleanolic acid in FLL extracts was quantified as $42.5 \mathrm{mg} / \mathrm{g}$, suggesting that the FLL extract was of high quality.

Body and Uterine Weights As shown in Fig. 2A, rats in all four experimental groups had similar initial body weights. Four weeks after operation, there was a significant increase in the body weight of the OVX rats $(p<0.01, v s$. sham). Treatment of OVX rats with $\mathrm{E}_{2}$ but not FLL significantly suppressed the increase in body weight associated with $\mathrm{E}_{2}$ deficiency and returned body weight to the level maintained by sham group four weeks after treatment. In addition, OVX caused significant atrophy of the uterus in rats as anticipated (Fig. 2B, $p<0.001 v s$. sham). $\mathrm{E}_{2}$ significantly increased uterine weight in OVX rats $(p<0.001)$ but the weight remained substantially lower than that of the sham rats $(p<0.001)$. In contrast, treatment of OVX rats with FLL extracts did not affect their uterine weight. 


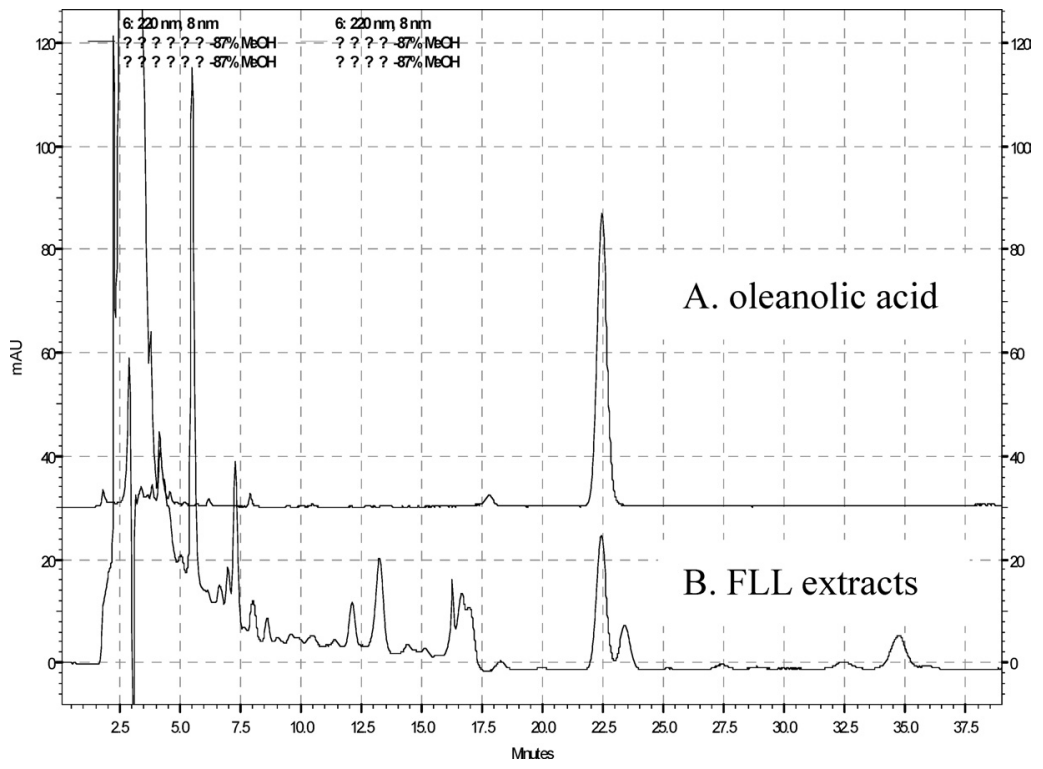

Fig. 1. Reverse-Phase HPLC for the Quantification of Oleanolic Acid in Fructus Ligustri Lucidi (FLL)

Peak eluting at $22.5 \mathrm{~min}$ is oleanolic acid. HPLC pictures for the oleanolic acid standard and FLL extracts are listed as upside and underside respectively.

$\mathbf{A}$

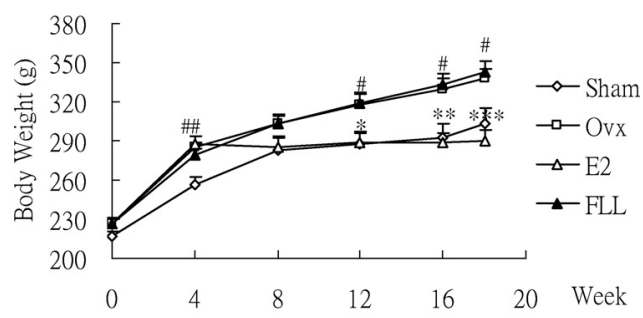

B

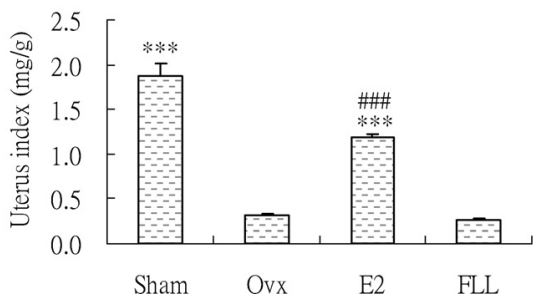

Fig. 2. Effects of Fructus Ligustri Lucidi (FLL) Extract on the Changes of Body Weight and Uterine Weight in Ovariectomized Rats

(A) Body weight was measured weekly during the experimental period in Sham group $(\diamond)$, OVX group $(\square)$, and OVX rats were orally administrated of $17-\beta$ estradiol at $2 \mu \mathrm{g} / \mathrm{kg} / \mathrm{d}(\triangle)$ and FLL extract at $550 \mathrm{mg} / \mathrm{kg} / \mathrm{d}(\Delta) 4$ weeks after operation and lasted for 14 weeks. (B) Uterine weight was measured after the rats were sacrificed and the uterus index was represented as uterine weight divide by body weight. Data are expressed as mean \pm S.E.M. $* p<0.05$, $* * p<0.01$, $* * * p<0.001$ vs. OVX group; $\# p<0.05, \# p<0.01, \# p<0.001$ vs. Sham group.

Serum and Urine Chemistries OVX significantly decreased serum calcium level (Table $1, p<0.05$ vs. sham) and increased urinary calcium level (Table $1, p<0.01$ vs. sham) in rats. $E_{2}$ significantly reversed the OVX-induced changes in serum and urinary calcium levels. Treatment of OVX rats with FLL extract also suppressed urinary calcium levels $(p<0.001 v s$. OVX rats). Serum and urinary phosphorus levels were similar in all groups. Both serum calcium and phosphorus levels in all groups were within the normal range of
Table 1. Effects of Fructus Ligustri Lucidi on Calcium and Phosphorus Level of Serum and Urine in Ovariectomized Rats

\begin{tabular}{llcll}
\hline \hline Group & \multicolumn{1}{c}{$\begin{array}{c}\mathrm{s}-\mathrm{Ca} \\
(\mathrm{mg} / \mathrm{dl})\end{array}$} & $\begin{array}{c}\mathrm{s}-\mathrm{P} \\
(\mathrm{mg} / \mathrm{dl})\end{array}$ & $\begin{array}{c}\mathrm{u}-\mathrm{Ca} / \mathrm{Cr} \\
(\mathrm{mg} / \mathrm{mg})\end{array}$ & $\begin{array}{c}\mathrm{u}-\mathrm{P} / \mathrm{Cr} \\
(\mathrm{mg} / \mathrm{mg})\end{array}$ \\
\hline Sham & $10.94 \pm 0.23^{*}$ & $5.72 \pm 0.20$ & $0.035 \pm 0.004^{* *}$ & $1.62 \pm 0.06$ \\
OVX & $10.35 \pm 0.13$ & $5.42 \pm 0.14$ & $0.068 \pm 0.007$ & $1.92 \pm 0.16$ \\
$\mathrm{E}_{2}$ & $11.18 \pm 0.23^{* *}$ & $5.63 \pm 0.29$ & $0.027 \pm 0.006^{* * *}$ & $1.78 \pm 0.23$ \\
FLL & $10.76 \pm 0.16$ & $5.83 \pm 0.31$ & $0.034 \pm 0.004^{* * *}$ & $1.91 \pm 0.07$ \\
\hline
\end{tabular}

Data are expressed as mean \pm S.E.M. Asterisks indicate difference from OVX group: ${ }^{*} p<0.05, * * p<0.01,{ }^{* * *} p<0.001$. s, serum; u, urine; Ca, calcium; P, phosphorus; Cr, creatinine.

the rats. ${ }^{17)}$

Bone Turnover Markers Serum osteocalcin (OCN, a specific product of the osteoblast) is a bone formation marker while urinary deoxypyridinoline (DPD, the breakdown product of collagen during bone resorption) is a bone resorption marker. OVX resulted in a significant increase in serum OCN and urinary DPD concentrations in OVX rats (Fig. 3, $p<0.01$ and $p<0.05 v s$. sham, respectively), suggesting an increase in bone turnover rate in OVX rats. Both FLL and $\mathrm{E}_{2}$ significantly suppressed the OVX-induced increase in serum OCN level $(p<0.05)$ in rats. In addition, FLL extract prevented the increase in urinary DPD levels in OVX rats $(p<0.05)$. This result indicated that FLL extract prevented the induction of high bone turnover associated with $\mathrm{E}_{2}$-deficiency in $\mathrm{OVX}$ rats.

Bone Calcium Content The effects of FLL extract on bone calcium content in rat tibia are summarized in Table 2. Ash weight of rat tibia was significantly lower in the OVX group ( $p<0.05, v s$. sham), suggesting that OVX induced the loss of inorganic mineral in bone. Treatment of OVX rats with FLL extract prevented the reduction of ash weight and calcium content of rat tibia in OVX rats $(p<0.01$ and $p<0.05$, vs. OVX, respectively). Thus, FLL extract could effectively prevent the loss of calcium from bone in $\mathrm{E}_{2}$-deficient rats. 
A

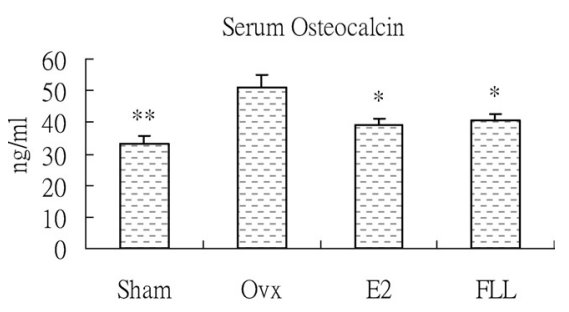

B

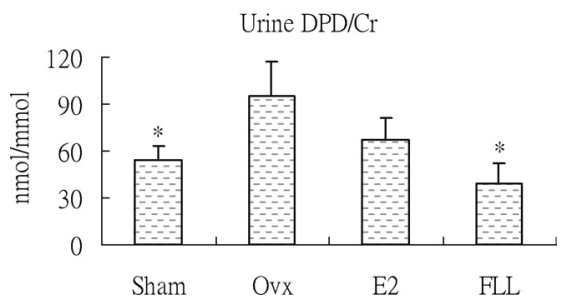

Fig. 3. Effects of Fructus Ligustri Lucidi (FLL) on Biochemical Markers of Bone Turnover in Ovariectomized Rats

(A) Serum osteocalcin is a sensitive marker of bone formation. (B) Urine DPD is a sensitive and specific marker of bone resorption. Data are expressed as mean \pm S.E.M $* p<0.05, * * p<0.01 v s$. OVX group.

Table 2. Effects of Fructus Ligustri Lucidi on Bone Mineral Content in Ovariectomized Rats

\begin{tabular}{lll}
\hline \hline Group & \multicolumn{1}{c}{$\begin{array}{c}\text { Ash weight } \\
(\mathrm{mg})\end{array}$} & $\begin{array}{c}\mathrm{Ca} / \text { Ash bone } \\
(\mu \mathrm{g} / \mathrm{mg})\end{array}$ \\
\hline Sham & $335.48 \pm 15.75^{*}$ & $334.90 \pm 1.84$ \\
OVX & $280.77 \pm 4.73$ & $334.74 \pm 1.73$ \\
$\mathrm{E}_{2}$ & $311.28 \pm 15.23$ & $338.29 \pm 1.43$ \\
FLL & $324.92 \pm 6.49^{* *}$ & $340.10 \pm 1.30^{*}$ \\
\hline
\end{tabular}

Data are expressed as mean \pm S.E.M. Asterisks indicate difference from OVX group: ${ }^{*} p<0.05,{ }^{* *} p<0.01$.

Table 3. Effects of Fructus Ligustri Lucidi on Calcium Balance and the Components of Calcium Balance in Ovariectomized Rats

\begin{tabular}{lcclll}
\hline \hline Group & $\begin{array}{c}\text { Food intake } \\
(\mathrm{g} / \mathrm{d})\end{array}$ & $\begin{array}{c}\text { Ca intake } \\
(\mathrm{mg} / \mathrm{d})\end{array}$ & $\begin{array}{c}\text { Urinary Ca } \\
(\mathrm{mg} / \mathrm{d})\end{array}$ & $\begin{array}{c}\text { Fecal Ca } \\
(\mathrm{mg} / \mathrm{d})\end{array}$ & $\begin{array}{c}\text { Ca balance } \\
(\mathrm{mg} / \mathrm{d})\end{array}$ \\
\hline Sham & $14.8 \pm 0.4$ & $148 \pm 4$ & $0.29 \pm 0.03^{* *}$ & $55.7 \pm 5.7^{* *}$ & $92 \pm 6^{* *}$ \\
OVX & $15.6 \pm 0.1$ & $156 \pm 1$ & $0.77 \pm 0.11$ & $87.3 \pm 7.3$ & $68 \pm 4$ \\
$\mathrm{E}_{2}$ & $14.2 \pm 0.7$ & $142 \pm 7$ & $0.22 \pm 0.09^{* *}$ & $48.3 \pm 4.5^{* *}$ & $94 \pm 9 * *$ \\
FLL & $14.9 \pm 0.5$ & $149 \pm 5$ & $0.46 \pm 0.07^{*}$ & $58.0 \pm 6.1^{* *}$ & $90 \pm 7^{* *}$ \\
\end{tabular}

Data are expressed as mean \pm S.E.M. Asterisks indicate difference from OVX group: ${ }^{*} p<0.05 ; * * p<0.01$.

Calcium Balance Study Figure 4 and Table 3 summarize the results from the calcium balance study. The net amount of calcium absorbed was calculated based on the difference between the amounts of calcium ingested in the diet and excreted in feces. Fecal calcium excretion was lower in the $\mathrm{E}_{2}$ and FLL group, and they absorbed and retained more calcium than the OVX group (Table 3). As shown in Fig. 4A, intestinal calcium absorption rate was significantly lower in OVX rats $(p<0.001, v s$. sham). Treatment of OVX rats with $\mathrm{E}_{2}$ or FLL extract significantly increased intestinal calcium absorption $(p<0.001$ and $p<0.01$ respectively). To calculate
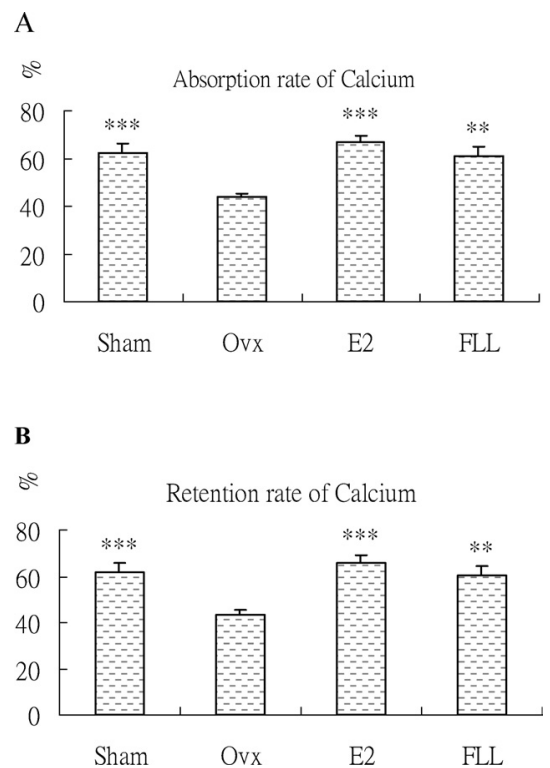

Fig. 4. Effects of Fructus Ligustri Lucidi (FLL) on Calcium Balance in Ovariectomized Rats

Calcium absorption was determined before the rats were sacrificed. (A) Absorption rate and (B) retention rate of calcium were calculated as methods. Data are expressed as mean \pm S.E.M. $* * p<0.01, * * * p<0.001 v s$. OVX group.

calcium retention rate, the amount of calcium loss from urine was deducted from that being absorbed into the body. As shown in Fig. 4B, OVX rats had the lowest calcium retention rate among all groups and treatment of OVX rats with either estradiol or FLL restored calcium retention rate to the levels achieved by the sham group.

\section{DISCUSSION}

The present study demonstrated that Fructus Ligustri Lucidi (FLL) could prevent high bone turnover and calcium loss caused by $E_{2}$ deficiency, without substantial effects on the uterus. Ovariectomy of young rats is a model for studying postmenopausal osteoporosis. ${ }^{18,19)}$ As expected, OVX animals in the present study exhibited all the characteristics associated with $\mathrm{E}_{2}$ deficiency, such as weight gain, negative calcium balance, high bone turnover and uterine atrophy. Although FLL has been widely used in China, limited data are available to substantiate their beneficial effects on osteoporosis. To our knowledge, the present study is the first demonstration of the protective effects of FLL against high bone turnover, loss of bone $\mathrm{Ca}$ and reduced $\mathrm{Ca}$ retention associated with $\mathrm{E}_{2}$ deficiency in OVX animals.

Our results confirmed the findings of others that the OVX rat model was characterized by high bone turnover rate ${ }^{20-22)}$ and that FLL extract could effectively prevent the OVXinduced increase in bone turnover. The increase in bone turnover was the result of increase in both bone formation and bone resorption associated with $\mathrm{E}_{2}$ deficiency. ${ }^{20)}$ Our study showed that serum OCN level, which is used as a clinical marker for detecting bone formation in vivo, was significantly increased in OVX rats. In addition, OVX caused a significant increase in urinary DPD excretion, a bone resorption marker. Treatment of OVX rats with FLL extract for 14 weeks significantly reduced serum OCN and urinary DPD levels, suggesting that FLL acts on bone as a potent inhibitor 
of high bone turnover. Whether the effects of FLL on bone turnover are primarily mediated by its actions on osteoblastic cells (bone formation) and/or osteoclastic cells (bone resorption) require further investigation. Regardless of its mechanism of action, the drastic decrease in rate of bone turnover provides a direct explanation for the observed increase in ash weight and calcium content of tibia in OVX rats treated with FLL extract.

It is of interest to note that FLL demonstrated selective estrogen-like effects on bone but without the detrimental stimulatory effects in the uterus. Recent reports ${ }^{23)}$ suggest that phytoestrogen, such as isoflavones, act as selective estrogen receptor modulator (SERM) because they exhibit estrogen activity in some tissues (bone), but act as estrogen antagonist in other tissues (breast, uterus). For example, daidzein had estrogenic effects at the bone without acting in an estrogenic manner at the uterus. ${ }^{24)}$ Moreover, uterotrophic effects may be correlated with the serum concentration of phytoestrogens. For instance, 10-fold higher level of serum genistein is required for this isoflavone to affect the uterus compared with that affecting bone tissue in OVX mice. ${ }^{25}$ Thus, it is possible that FLL might behave like phytoestrogens that possess selective activities toward bone tissue and uterus.

Treatment with FLL extract also increased intestinal calcium absorption and calcium retention that were otherwise reduced in OVX rats. It is well known that $\mathrm{E}_{2}$ is essential for intestinal calcium absorption and $\mathrm{E}_{2}$ withdrawal in addition to having a direct effect on the skeleton, perpetuating calcium deficiency by decreasing calcium absorption and increasing calcium excretion in human and animals. ${ }^{26-29)}$ Recent studies showed that the stimulation of intestinal calcium absorption by estrogen was estrogen receptor (ER) dependent and that ERs were present in rat duodenal cells. ${ }^{30,31)}$ The results in the present study confirmed that administration of 17beta-estradiol in OVX rats could improve the intestinal calcium absorption and retention rates. As FLL extract can also improve calcium balance in OVX rats, it is of interest to further determine whether FLL behaves like phytoestrogens in stimulating intestinal calcium absorption in OVX rats.

The observed effects of FLL on calcium homeostasis could also be mediated by its modulation of calcium-regulating hormones. It is well known that parathyroid hormone and 1,25-dihydroxyvitamin D3 $\left[1,25(\mathrm{OH})_{2} \mathrm{D}_{3}\right]$ are important hormones involved in regulating intestinal calcium absorption. ${ }^{32)}$ The parathyroid glands sense the serum calcium level, and secrete parathyroid hormone (PTH) if it becomes too low, for example, when dietary calcium intake is inadequate. PTH stimulates the activity of the 1-hydroxylase in the kidney, resulting in increased production of calcitriol, the biologically active form of vitamin D3. ${ }^{33)}$ Increased calcitriol production restores normal serum calcium levels in three different ways: 1) by activating the vitamin D-dependent calcium transport system in the small intestine, increasing the absorption of dietary calcium, 2) by increasing the mobilization of calcium from bone into the circulation, and 3 ) increasing the reabsorption of calcium by the kidneys. PTH is also required to increase bone calcium mobilization and calcium reabsorption by the kidneys. Thus, it is possible that the actions of FLL could be mediated by alteration of PTH synthesis or secretion, by alteration of the biosynthesis of $1,25(\mathrm{OH})_{2} \mathrm{D}_{3}$ or by increasing the intestinal sensitivity to vitamin $\mathrm{D}$ via modulat- ing the level of vitamin D receptor expression. Further study will be needed to determine the effects of FLL extract on vitamin D metabolism.

Fructus Ligustri Lucidi is a plant widely distributed in the southern China and manifests its therapeutic actions in kidney deficiency. Noting the TCM theory that "kidney dominates bone," we investigated whether FLL had any beneficial effect on bone and mineral metabolism in osteoporotic rats. Our results clearly demonstrate that administration of FLL extract inhibited high bone turnover, elevated intestinal calcium absorption and prevented calcium loss in young ovariectomized rats. Our study provides evidence that FLL could be a potential candidate for the treatment of postmenopausal osteoporosis. The molecular and cellular mechanisms by which FLL extract exert these effects in vivo, remain to be clarified.

Acknowledgements The authors would like to thank the State Key Laboratory of Chinese Medicine and Molecular Pharmacology for providing the support in carrying out this study. This work was supported by the Areas of Excellence Scheme Established under the University Grants Committee of the Hong Kong Special Administrative Region, China (AOE/P-10/01) and the Earmarked Research Grant Council (B-Q767, 3-ZF89), HKSAR.

\section{REFERENCES}

1) Kaplan B., Hirsch M., Clin. Exp. Obstet. Gynecol., 31, 251-255 (2004).

2) Stevenson J. C., Maturitas, 51, 113-126 (2005).

3) Prelevic G. M., Kocjan T., Markou A., Minerva. Endocrinol., 30, 2736 (2005)

4) Wiseman R. A., J. Clin. Epidemiol., 57, 766-772 (2004).

5) Davison S., Davis S. R., Clin. Endocrin., 58, 249-261 (2003).

6) Shi H. L., Infor. Trad. Chin. Med., 21, 59-60 (2004).

7) Pharmacopoeia Commission of the Ministry of Public Health, "Chinese Pharmacopoeia, Part I," 2005 edition, Chemical Industrial Press, Beijing, China, 2005.

8) Liang S. M., Tonic Methods, Zhong Yao Fang Ji Xue, Guangdong, Guangdong Science \& Technology Press, 1991, p. 656.

9) Liu H., Shi Y., Wang D., Yang G., Yu A., Zhang H., J. Pharm. Biomed. Anal., 32, 479-485 (2003).

10) Li M. L., Lau M. L., Fung F. H., China J. Chinese Mat. Med., 19, 504-506 (1994)

11) Wang T., Zeng Y., Xu M., Xie J. H., Trad. Chin. Drug Res. Clin. Pharm., 14, 403- 405 (2003).

12) Huang W., Yang Y. F., Modern J. Integ. Trad. Chin. Wester. Med., 12, $772-774$ (2003)

13) Bown D., "Encyclopaedia of Herbs and Their Uses," Dorling Kindersley, London, 1995.

14) Hao S. C., Bi P., Yu K., Sun J. H., Ding L. Q., J. Tianjin Normal University (Natural Science Edition), 17(3), 49-52 (1997).

15) National Research Council, "Guide for the Care and Use of Laboratory Animals," National Academy Press, Washington D.C., 1996.

16) Iwamoto J., Yeh J. K., Takeda T., Ichimura S., Sato Y., Bone, 33, 557566 (2003).

17) Kuniyoshi M., Tetsuo I., J. Bone Miner. Metab., 18, 194-207 (2000).

18) Kalu D. N., Bone Miner., 15, 175-191 (1991).

19) Thompson D. D., Simmons H. A., Pirie C. M., Ke K. Z., Bone, 17, 125S-133S (1995).

20) Shiraishi A., Higashi S., Masaki T., Saito M., Ito M., Ikeda S., Nakamura T., Calcif. Tissue Int., 71, 69-79 (2002).

21) Picherit C., Bennetau P. C., Chanteranne B., Lebecque P., Davicco M. J., Barlet J. P., Coxam V., J. Nutr., 131, 723-728 (2001).

22) da Paz L. H., de Falco V., Teng N. C., dos Reis L. M., Pereira R. M., Jorgetti V., Braz. J. Med. Biol. Res., 34, 1015-1022 (2001).

23) Setchell K. D., J. Am. Coll. Nutr., 20, 354S-362S (2001). 
24) Debbie F., Wendy E. W., Bone, 35, 489-497 (2004).

25) Yoshiko I., Naoko A., Xinxiang W., Jian W., Keizo U., Chisato M., Akiharu T., Sachie I., Biochem. Biophys. Res. Commun., 274, 697-701 (2000).

26) Fujita T., Calcif. Tissue Int., 58, 215 (1996).

27) Loughlin O. P. D., Morris H. A., J. Physiol., 511, 313-322 (1998).

28) Holzherr M. L., Retallack R. W., Gutteridge D. H., Price R. I., Faulkner D. L., Wilson S. G., Will R. K., Stewart G. O., Stuckey B. G., Prince R. L., Criddle R. A., Kent G. N., Bhagat C. I., Dhaliwal S. S., Jamrozik K., Osteoporos. Int., 11, 43-51 (2000).
29) Kalu D. N., Orhii P. B., Calcif. Tissue Int., 65, 73-77 (1999).

30) Bolscher M., Netelenbos J. C., Barto R., Buuren L. M., Vijgh W. J., J. Bone Miner. Res., 14, 1197-1202 (1991).

31) Chen C., Noland K. A., Kalu D. N., Mech. Ageing Dev., 99, 109-122 (1997).

32) Mitamura R., Hara H., Aoyama Y., Biosci. Biotechnol. Biochem., 68, 384-389 (2004).

33) Murayama A., Takeyama K. I., Kitanaka S., Kodera Y., Kawaguchi Y., Hosoya T., Kato S., Endocrinology, 140, $2224-2231$ (1999). 